\title{
Comparison of the Objective Measures and Behavioral Responses in Cochlear Implant Users Implanted with CI422: A Preliminary Study
}

\author{
Merve Özbal Batuk ${ }^{1}$ (iD, Betül Çiçek Çınar² ${ }^{2}$
}

\begin{abstract}
Objectives: During the programming of the cochlear implant (CI) various fitting methods can be used such as electrically stapedial reflex thresholds (ESRT), electrically compound action potential (ECAP) thresholds and behavioral methods with visual loudness scales. The aim of the present study is to investigate the relationship between the eSRT and eCAP thresholds with the behavioral comfortable levels in CI users implanted with CI422. Materials and Methods: Ten CI users included in the study with the mean age of 20.47. Five electrodes were selected from different regions of cochlea for comparison. For these five electrodes, behavioral C (comfortable) levels were determined with Custom Sound ${ }^{\circ}$ 5.2 Fitting Software and eCAP and eSRT thresholds were obtained with Custom Sound $\circledast$ EP 5.2.

Results: Correlation analysis was done between behavioral C levels and eSRT/eCAP separately. For E22, behavioral $\mathrm{C}$ level was positively correlated with eSRT $(\mathrm{r}=.772 ; \mathrm{p}=.009 ; \mathrm{p}<.01)$. However, there was no correlational relation with eCAP. For E1, behavioral $\mathrm{C}$ level was positively correlated with both eSRT ( $\mathrm{r}=0.785$; $\mathrm{p}=.007 ; \mathrm{p}<.01)$ and eCAP $(\mathrm{r}=0.812 ; \mathrm{p}=.004 ; \mathrm{p}<.01)$. There was not any correlational relation between eSRT levels and eCAP thresholds except for E1. Behavioral $\mathrm{C}$ levels was compared with ECAP and ESRT with related-samples Wilcoxon test. The eSRT levels were found significantly higher than the behavioral C levels for all electrodes $(\mathrm{p}<.05)$.

Conclusion: This study is the first study that investigates the objective measures and behavioral method in CI users with Cochlear CI422 implant. Both eCAP and eSRT levels can be used in CI fitting but eSRT levels are significantly higher than the behavioral $\mathrm{C}$ levels. Especially in the basal portion of the cochlea, the C levels are correlated both with eCAP and eSRT levels. It is possible to use both objective thresholds in adjustment of the C levels in basal region in patients with CI422 implant. In order to provide adequate stimulation, the combination of the objective measures and behavioral methods was considered to be the best option.
\end{abstract}

Keywords: hearing loss, cochlear implants, cochlear implantation, electric stimulation, auditory threshold

\footnotetext{
${ }^{1}$ Merve Özbal Batuk (Corresponding Author). Hacettepe University, Faculty of Health Sciences, Department of Audiology, 03123051667. e-mail: merveozbal@ @otmail.com

${ }^{2}$ Betül Çiçek Çınar. Hacettepe University, Faculty of Health Sciences, Department of Audiology, 03123051667. e-mail: betulcicek01@gmail.com
} 


\section{Introduction}

Cochlear implant (CI) is a surgically implanted system that electrically stimulates the auditory nerve in individuals with severe to profound hearing loss and who cannot benefit from conventional hearing aids (Wolfe et al., 2016). After surgery, each channel of the CI electrode stimulates the auditory nerve and each patient needs individualized programming of the CI system. The minimum (threshold, T level) and the maximum (comfortable, $\mathrm{C}$ level) electrical stimulation levels are adjusted by an audiologist in the programming sessions (Andrade et al., 2014).

During the programming of the CI, various fitting methods can be used such as behavioral methods with visual loudness scales and objective measures like electrically stapedial reflex thresholds (eSRT), electrically compound action potential (eCAP) thresholds and electrically auditory brainstem response (eABR).

In adult population, it is easy to use behavioral methods in programming but in pediatric CI users, the behavioral responses are not reliable due to the supra-threshold responses of the children. On the other, using the behavioral method and the objective measures together are thought to be more effective in adaptation of the CI and in providing adequate electrical stimulation (Andrade et al., 2014).

The eCAP measurements are early neural responses that show the synchronizing neural firing of the spiral ganglions in 0-2 msec (Caner, Olgun, Gultekin, and Balaban, 2007). Although eCAP measures are the easiest objective electrical methods without any equipment other than CI fitting software, it is considered as a poor predictor of the behavioral thresholds (Wolfe et al., 2016).

The electrically stapedius reflex threshold (eSRT) measures are another objective measurement technique that show the upper stimulation levels with the stimulation of the stapes muscle (Wolfe et al., 2016). The eSRT can be used in the determination of the maximum electrical stimulation level both in adults and children (Gordon, Papsin, and Harrison, 2004). However, the main disadvantage of the ESRT is that reflex cannot be achieved in many users and is difficult to use in young children (Kosaner, Spitzer, Bayguzina, Gultekin, and Behar, 2018).

In order to record eCAP successfully, adequate neural synchronization is required with slow speed stimulation. In contrast, high speed modulated stimuli are used during the stimulation with CI. It is discussed whether it is a suitable method to determine the correlation between ECAP thresholds and behavioral stimuli due to the use of the different speed of stimuli 
in recording. On the contrary, ESRT is not affected by the same recording restrictions as the eCAP measurements. Stimuli similar to the electrical stimuli used in the programming can be utilized to obtain the ESRT response (Wolfe et al., 2016; Overstreet, 2004).

Although many studies investigated the correlation between the objective measures and the behavioral method in different types of implants (Smoorenburg, Willeboer, and van Dijk, 2002; Wolfe et al., 2016; Overstreet, 2004; Walkowiak et al., 2011; Kosaner et al., 2018), there is no standardization regarding the use of these measures during the CI programming sessions. To our knowledge, there is not any study showing the result of the correlation between the behavioral stimulation levels and the objective measures in patients with Cochlear Nucleus CI422 electrode. The aim of the present study is to investigate the relationship between the eSRT and eCAP thresholds with the behavioral comfortable levels in CI users implanted with CI422.

\section{Materials and Methods}

The study conducted at Hacettepe University Audiology Department. Subjects and their parents were informed about the content of the study and their consent forms were filled out.

\section{Participants}

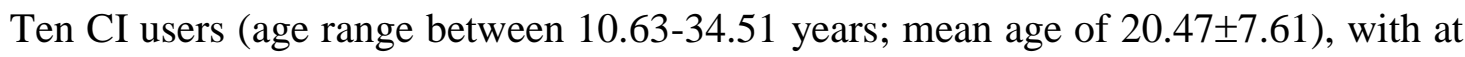
least one year of experience with CI were included to the current study. Subjects who has active middle or outer ear pathology, has less than 20 active electrodes, has a partial insertion of the electrode array, has an etiology of inner ear malformation, cochlear nerve deficiency or otosclerosis were excluded. All subjects received their CI in Hacettepe University Hospital, Turkey. The demographic characteristics of the subjects were shown in Table 1.

Table 1. Demographic characteristics of subjects

\begin{tabular}{ccccccc}
\hline Subject & Gender & $\begin{array}{c}\text { Age at CI } \\
\text { (year) }\end{array}$ & $\begin{array}{c}\text { Duration of CI use } \\
\text { (year) }\end{array}$ & $\begin{array}{c}\text { Implant } \\
\text { Side }\end{array}$ & $\begin{array}{c}\text { Type of } \\
\text { Implant }\end{array}$ & $\begin{array}{c}\text { Type of } \\
\text { SP }\end{array}$ \\
\hline S1 & F & 28.61 & 1.00 & R & CI422 & Kanso \\
S2 & M & 16.16 & 3.16 & R & CI422 & Kanso \\
S3 & M & 15.10 & 1.00 & L & CI422 & CP910 \\
S4 & F & 16.94 & 1.86 & R & CI422 & Kanso \\
S5 & F & 12.46 & 1.62 & R & CI422 & Kanso \\
S6 & F & 16.90 & 1.95 & R & CI422 & Kanso \\
S7 & M & 24.49 & 1.11 & R & CI422 & Kanso \\
S8 & F & 10.93 & 2.17 & R & CI422 & Kanso \\
S9 & F & 34.51 & 1.65 & R & CI422 & CP910 \\
S10 & F & 10.63 & 2.83 & R & CI422 & CP910 \\
\hline
\end{tabular}

F: female; M: male; CI: cochlear implant; SP: sound processor 


\section{Methods}

Intracochlear electrode number is 22 in Cochlear Nucleus ${ }^{\circledR}$ Implant Systems. Electrode22 (E22) is located in the apical, whereas the E1 is located in the basal portion of the cochlea. In this study, five electrodes (E22 or E21, E16, E11, E6 and E1 or E2) were selected from different regions of cochlea for comparison. In order to evaluate the responses obtained from the apical, middle and basal regions of the cochlea, these electrodes were selected as these five electrodes were measured during routine control. For these five electrodes, behavioral C levels were determined with Custom Sound ${ }^{\circledR}$ 5.2 Fitting Software and eCAP and eSRT thresholds were obtained with Custom Sound $®$ EP 5.2.

To determine the behavioral $\mathrm{C}$ levels, visual loudness scales were used. For each individual electrode, stimulations levels start just below available $\mathrm{C}$ level and increased until the users fell it is loud enough. In eCAP measurement, the starting point was determined as 100CL (default). The stimulation level was automatically increased (Auto Neural Response Telemetry, Auto NRT Module) with 5CL until detectable eCAP threshold. For eSRT measurements, Interacoustics TITAN tympanometer was used in the reflex decay mode and a plastic probe was located in the entire of the outer ear canal on the opposite side of the CI. Normal middle ear pressure is required and the contralateral side to the CI was selected for all subjects. Similarly with eCAP, the starting level was 100CL and it was increased with 5CL until detectable eSRT level.

A statistical analysis was performed using SPSS 23 software. The comparisons between C levels, eCAP thresholds and eSRT levels were examined using Wilcoxon test. The relationship between the $\mathrm{C}$ levels, eCAP thresholds and eSRT levels were analyzed using Pearson Correlations. The statistical significance was set as $\mathrm{p}<.05$.

\section{Results}

\section{Descriptive Statistics}

There were seven female and three male CI users. The mean age at implantation was 18,67 with 7,79 standard deviation. The mean for CU usage was 1,83 years with 0.79 standard deviation. All participants implanted with CI422. Two of them use CP910 and others use Kanso speech processor. Just one subject was implanted on the left side, remaining nine was implanted on the right side. 


\section{Correlations}

Correlations analysis was done between behavioral $\mathrm{C}$ levels and eSRT or eCAP separately. For E22, behavioral C level was positively correlated with eSRT ( $r=.772 ; p=.009$; $\mathrm{p}<.01)$, however, there was no correlational relation with eCAP $(r=.435 ; \mathrm{p}=.208 ; \mathrm{p}>.05)$. For $\mathrm{E} 1$, behavioral C level was positively correlated with both eSRT $(r=0.785 ; p=.007 ; p<.01)$ and eCAP $(r=0.812 ; \mathrm{p}=.004 ; \mathrm{p}<.01)$. However, for electrodes 16 and 11, there was no correlation between behavioral $\mathrm{C}$ levels and $\operatorname{eSRT}$ or eCAP ( $\mathrm{p}>.05$ ). Additionally, there was not any correlational relation between eSRT and eCAP except E1 ( $\mathrm{r}=.673 ; \mathrm{p}=.033 ; \mathrm{p}<.05)$.

\section{Comparisons}

Behavioral C levels was compared with ECAP and ESRT with related-samples Wilcoxon test. Behavioral $\mathrm{C}$ levels for all electrodes are statistically significantly different from eSRT levels ( $\mathrm{p}=.007$ for $\mathrm{E} 22 ; \mathrm{p}=.009$ for $\mathrm{E} 16 ; \mathrm{p}=.013$ for $\mathrm{E} 11 ; \mathrm{p}=.012$ for $\mathrm{E} 6$ and $\mathrm{E} 1 ; \mathrm{p}<.05$ ). However, behavioral C levels are not statistically significantly different from eCAP levels for $\mathrm{E} 22(\mathrm{p}=.285), \mathrm{E} 16(\mathrm{p}=.285), \mathrm{E} 6(\mathrm{p}=.051)$ and $\mathrm{E} 1(\mathrm{p}=.51)(\mathrm{p}>.05)$ but it was significant for $\mathrm{E} 11$ $(\mathrm{p}=.008 ; \mathrm{p}<.05)$. Figure 1 shows the mean levels of behavioral $\mathrm{C}$ levels, ECAP and ESRT in current units. Also, no significant difference was observed between the ECAP thresholds and ESRT levels $(\mathrm{p}<.05)$.

Figure 1. Schematic view of the mean behavioral C levels, ECAP thresholds and ESRT levels

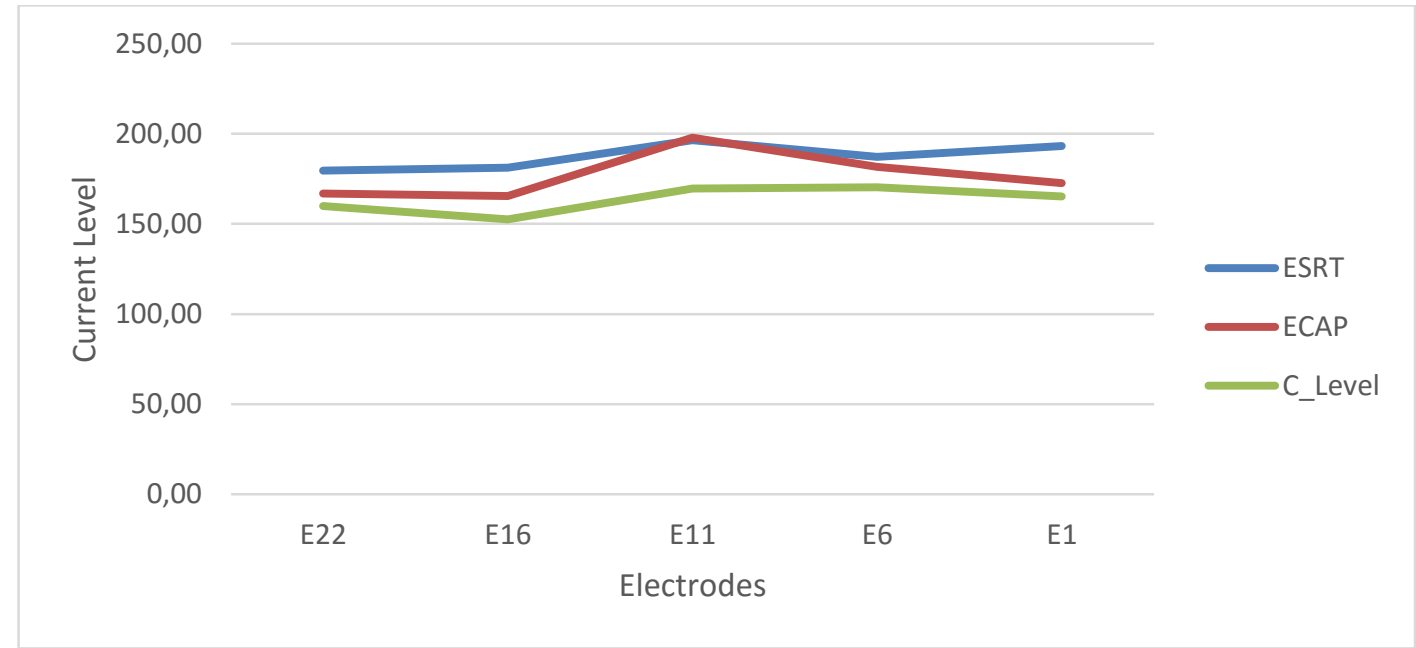

\section{Discussion and Conclusion}

The results of the present study demonstrate a significant correlation between eSRT/eCAP thresholds and C levels in basal electrode region whereas a significant correlation was only observed between only eCAP thresholds and C levels in apical electrode region. On 
the contrary, no significant relationship was observed between not only eSRT levels but also eCAP thresholds and $\mathrm{C}$ levels in midportion electrodes. According to our findings, eSRT levels were found higher than the $\mathrm{C}$ levels in all electrode regions.

The eSRT levels were not found statistically higher than the eCAP thresholds in the current study. Kosaner et al. (2018) and Walkowiak et al. (2011) reported contrary results. Kosaner et al. evaluated the eSRT values and eCAP thresholds in children with Med-El CI and found out that eCAP thresholds are significantly lower than the eSRT levels (Kosaner et al., 2018). Walkowiak et al. (2011) evaluated the eSRT levels and eCAP thresholds in 16 adults and 14 children with Med-El Pulsar CI100. They reported that eCAP thresholds are significantly lower than the $\mathrm{C}$ levels and the $\mathrm{C}$ levels are corelated both with eSRT levels and eCAP thresholds in adults (Walkowiak et al., 2011). The exact explanation is that the number of participants in our study is very small. Despite the eSRT levels are higher than the eCAP thresholds, the difference is not statistically significant.

The CI422 implant is inserted to the lateral wall which was far away from modiolus. Even though, the $\mathrm{C}$ levels was similar to the perimodiolar electrodes, eCAP thresholds was found higher in lateral wall electrode side in patients with sequential bilateral CI (Park et al., 2017). A recent study published by the authors showed that eCAP thresholds are similar in both perimodiolar and lateral wall electrodes (Batuk et al., 2019). Despite eSRT levels were significantly higher than $\mathrm{C}$ levels, eCAP thresholds was not found different from $\mathrm{C}$ levels in the current study.

Our results showed that there is a strong positive correlation between eCAP thresholds and C levels only in E1. It is hard to explain whether this happens on a single electrode. Many studies have shown that eCAP is not a good predictor in the determination of the electrical stimulation levels in patients with CI (Wolfe et al., 2016; Smoorenburg et al., 2002).

Even though eSRT was known as the best method the determine the maximum stimulation levels in CI. Not only providing adequate stimulation but also reducing the incidence of the non-auditory stimulation such as facial twitching, eye blinking (Gordon et al.,2004; Wolfe et al., 2016). Despite the advantages of eSRT, the incidence of the use of eSRT in the clinical settings was found as 14\% (Vaerenburg et al., 2014). The main reason why eSRT is less preferred is that it cannot be observed in all subjects. In previous studies the percentage of the observation of eSRT in CI users ranged from 67\% to 90\% (Battmer, Laszig, and Lehnhardt, 1990; Gordon et al., 2004; Cinar, Atas, G. Sennaroglu, and L. Sennaroglu, 2011). In the study of Cinar et al. (2011), it was found that the most reliable objective test method is eABR when 
compared with eCAP and eSRT in CI users with inner ear malformation. Nevertheless, eCAP was observed in $74 \%$ of the subjects with normal cochlea whereas eSRT was obtained $90 \%$ of the same control group (Cinar et al., 2011). In our small cohort of subjects, we observed both eSRT and eCAP thresholds in all participants.

Many papers compared the objective measures with behavioral methods, in Med-El implant, Advanced Bionics implant or in different type of Cochlear ${ }^{\mathrm{TM}}$ implant electrodes. Our study is the first study that investigates the objective measures and behavioral method in adults with CI422 implants. Both eCAP and eSRT can be used in CI fitting but eSRT levels are significantly higher than the behavioral $\mathrm{C}$ levels. Especially in the basal portion of the cochlea, the $\mathrm{C}$ levels are correlated both with eCAP and eSRT levels. It is possible to use both objective thresholds in adjustment of the $\mathrm{C}$ levels in basal region in patients with CI422 implant. In order to provide adequate stimulation, the combination of the objective measures and behavioral methods was considered to be the best option. The main limitation of the present study was the limited number of subjects. Our study investigated the relationship between objective measures and behavioral method in adults with CI422 implant and exhibited the preliminary results of this investigation.

\section{Acknowledgement}

The authors would like to thank Prof. Gonca Sennaroglu and Aud. Aysun Parlak Kocabay for the support in the study conception and design.

\section{Financial Support}

The authors have no financial support to declare.

\section{Conflict of Interest}

The authors have no conflicts of interest to declare. 


\section{References}

Andrade, K.C.L., Leal, M.C, Muniz, L.F., Menezes, P.L., Albuquerque, K.M.G. \& Carnaúba A.T.L. The importance of electrically evoked stapedial reflex in cochlear implant. Brazilian Journal of Otorhinolaryngology. 2014; 80:68-77.

Battmer, R., Laszig, R. \& Lehnhardt, E. (1990) Electrically elicited stapedius reflex in cochlear implant patients. Ear and Hearing. 1990 (11),370-374.

Batuk, M.O., Cinar, B.C., Zeren, E., Bayulgen, Ö., Dusunmez, İ. \& Sennaroglu, G. (2019). Evaluation of ECAP thresholds, $\mathrm{T}$ and $\mathrm{C}$ levels in children with sequential bilateral cochlear implants, Annals of Medical Research, 2019, 26(7): 1372-1377.

Caner, G., Olgun, L., Gultekin, G. \& Balaban, M. (2007). Optimizing fitting in children using objective measures such as neural response imaging and electrically evoked stapedius reflex threshold. Otology and Neurotology. 2007 (28), 637-640.

Cinar, B.C., Atas, A., Sennaroglu, G. \& Sennaroglu, L. (2011). Evaluation of objective test techniques in cochlear implant users with inner ear malformations. Otology and Neurotology, 2011(32),1065-1074.

Gordon, K.A., Papsin, B.C. \& Harrison, R.V. (2004). Toward a battery of behavioral and objective measures to achieve optimal cochlear implant stimulation levels in children. Ear and Hearing. 2004 (25), 447-463.

Kosaner, J., Spitzer, P., Bayguzina, S., Gultekin, M. \& Behar, L.A. (2018) Comparing eSRT and eCAP measurements in pediatric MED-EL cochlear implant users, Cochlear Implants International, 19 (3), 153-161, DOI: 10.1080/14670100.2017.1416759.

Overstreet, E.H. (2004). New objective measurement techniques and their relationship to HiResk program settings. International Congress Series, 1273 (2004), 35-39.

Park, L.R., Teagle, H.F., Brown, K.D., Gognon, E.B., Woodard, J.S. \& Buchman, C.A. (2017). Audiological outcomes and map characteristics in children with perimodiolar and slim straight array cochlear implants in opposite ears. Otology Neurotology, 2017;38 (9): 320-326.

Smoorenburg, G. F., Willeboer, C. \& van Dijk, J. E. (2002). Speech perception in nucleus CI24M cochlear implant users with processor settings based on electrically evoked compound action potential thresholds. Audiology Neurootology, 7, 335-347.

Vaerenberg, B., Smits, C., De Ceulaer, G., Zir, E., Harman, S. \&Jaspers, N. et al. (2014). Cochlear implant programming: a global survey on the state of the art. ScientificWorld Journal, 2014, 112, ID: 501738.

Walkowiak, A., Lorens, A., Polak, M., Kostek, B., Skarzynski, H. \& Szkielkowska, A. et al. (2011). Evoked stapedius reflex and compound action potential thresholds versus most comfortable loudness level: assessment of their relation for charge-based fitting strategies in implant users. Journal of Oto-Rhino-Laryngology, Head and Neck Surgery, 2011(73), 189-195.

Wolfe, J., Gilbert, M., Schafer, E., Litvak, L.M., Spahr, A.J.\& Saoji, A. et al. (2016). Optimizations for the electrically-evoked stapedial reflex threshold measurement in cochlear 1mplant recipients, Ear and Hearing, 38(2): 255-261. 